ARTICLE OPEN

\title{
Theoretical potential for low energy consumption phase change memory utilizing electrostatically-induced structural phase transitions in $2 \mathrm{D}$ materials
}

\author{
Daniel A. Rehn (iD) ${ }^{1}$, Yao $\mathrm{Li}^{2}$, Eric Pop ${ }^{3}$ and Evan J. Reed ${ }^{4}$
}

Structural phase-change materials are of great importance for applications in information storage devices. Thermally driven structural phase transitions are employed in phase-change memory to achieve lower programming voltages and potentially lower energy consumption than mainstream nonvolatile memory technologies. However, the waste heat generated by such thermal mechanisms is often not optimized, and could present a limiting factor to widespread use. The potential for electrostatically driven structural phase transitions has recently been predicted and subsequently reported in some two-dimensional materials, providing an athermal mechanism to dynamically control properties of these materials in a nonvolatile fashion while achieving potentially lower energy consumption. In this work, we employ DFT-based calculations to make theoretical comparisons of the energy required to drive electrostatically-induced and thermally-induced phase transitions. Determining theoretical limits in monolayer MoTe ${ }_{2}$ and thin films of $\mathrm{Ge}_{2} \mathrm{Sb}_{2} \mathrm{Te}_{5}$, we find that the energy consumption per unit volume of the electrostatically driven phase transition in monolayer $\mathrm{MoTe}_{2}$ at room temperature is $9 \%$ of the adiabatic lower limit of the thermally driven phase transition in $\mathrm{Ge}_{2} \mathrm{Sb}_{2} \mathrm{Te}_{5}$. Furthermore, experimentally reported phase change energy consumption of $\mathrm{Ge}_{2} \mathrm{Sb}_{2} \mathrm{Te}_{5}$ is $100-10,000$ times larger than the adiabatic lower limit due to waste heat flow out of the material, leaving the possibility for energy consumption in monolayer $\mathrm{MoTe}_{2}$-based devices to be orders of magnitude smaller than $\mathrm{Ge}_{2} \mathrm{Sb}_{2} \mathrm{Te}_{5}$-based devices.

npj Computational Materials (2018)4:2 ; doi:10.1038/s41524-017-0059-2

\section{INTRODUCTION}

Phase-change materials have long been studied for their applications in thermal energy storage ${ }^{1}$ and information storage. ${ }^{2,3}$ For thermal energy storage, water is one of the most common phase-change substances due to its large heat of fusion. For information storage, the most widely used phase-change material is $\mathrm{Ge}_{2} \mathrm{Sb}_{2} \mathrm{Te}_{5}$ alloy, ${ }^{2}$ which can exist in both amorphous and crystalline forms, each having different optical and electrical properties. ${ }^{4,5}$ The fast and reversible phase transition in $\mathrm{Ge}_{2} \mathrm{Sb}_{2} \mathrm{Te}_{5}$ alloys can be achieved by Joule heating, ${ }^{5}$ and this process can be employed in nonvolatile phase-change memory with lower programming voltages and higher memory densities than mainstream nonvolatile memory technologies. ${ }^{2,3,6,7}$

Some layered and two-dimensional (2D) materials including $\mathrm{MoTe}_{2}$ have been reported or predicted to exhibit structural phase changes during chemical processes, ${ }^{8,9}$ temperature changes, ${ }^{10-14}$ and tensile strains. ${ }^{15,16}$ Some single layer transition metal dichalcogenide (TMD) monolayers, including monolayer $\mathrm{MoTe}_{2}$, can exist in a semiconducting trigonal prismatic state found in the bulk $2 \mathrm{H}$ structure, a semimetallic distorted octahedral state found in the bulk $1 \mathrm{~T}^{\prime}$ monoclinic and orthorhombic (sometimes referred to as $T_{\mathrm{d}}$ ) phases, and possibly other structures. These are structures of the individual single layers, and not merely associated with different stacking sequences of the bulk material.
The monoclinic and orthorhombic phases exhibit similar single layer structure and are distinguished by slightly different stacking arrangements.

Our recent theoretical work suggests that a structural phase transition can be driven in specific monolayer materials through electrostatic gating, ${ }^{17}$ including monolayer $\mathrm{MoTe}_{2}$ and potentially $\mathrm{TaSe}_{2}$. This transition has been reported to occur in subsequent experiments employing ionic liquids to achieve the requisite charge densities. ${ }^{18}$ In the case of monolayer $\mathrm{MoTe}_{2}$, the transition is between the single layer structures of the $2 \mathrm{H}$ and $1 \mathrm{~T}^{\prime}$ phases. The switching between these two different phases can be achieved through electrostatic gating, allowing excess charge to enter or leave the monolayer. ${ }^{17}$ Note that the transition is driven by the addition or removal of charge from the material rather than the electric field associated with gating, i.e., a charge neutral monolayer in an applied field will not necessarily exhibit the effect. This mechanism is fundamentally distinct from thermally driven phase transitions. One can also envision devices that manipulate both temperature and charge independently to efficiently manipulate the stable phase and kinetics of the transition.

Other studies have reported that transitions between charge density wave $(\mathrm{CDW})$ states on the $(001)$ surface of layered $\mathrm{TaS}_{2}$ occur with changes in temperature. ${ }^{19,20}$ In addition, electrostatic gating has been reported to exhibit some control over the

\footnotetext{
'Department of Mechanical Engineering, Stanford University, Stanford, CA 94305, USA; ${ }^{2}$ Department of Applied Physics, Stanford University, Stanford, CA 94305, USA: ${ }^{3}$ Department of Electrical Engineering, Stanford University, Stanford, CA 94305, USA and ${ }^{4}$ Department of Materials Science and Engineering, Stanford University, Stanford, CA 94305, USA

Correspondence: Evan J. Reed (evanreed@stanford.edu)

Daniel A. Rehn and Yao Li contributed equally to this work.
}

Received: 14 September 2017 Revised: 6 December 2017 Accepted: 14 December 2017 Published online: 17 January 2018 
transition temperatures. ${ }^{21}$ While these CDW transitions are largely electronic rather than structural, their existence, and manipulation in layered materials suggests there may also be other materials and mechanisms for nonvolatility in very thin materials. However, practical widespread use of these CDW transitions for electronic applications may be limited due to the low transition temperatures between phases, which usually occur below room temperature.

For nonvolatile information storage applications, heat dissipation to the surroundings produced during the phase change can place an undesirable practical limit in achieving high device densities on a chip. ${ }^{22}$ Research into lower energy consumption phase change memory is active and ongoing, ${ }^{23-25}$ with efforts to scale down device sizes. As the spatial dimension of thermally controlled phase change materials decreases, the thermal resistances of adjacent interfaces play a dominant role in energy consumption, ${ }^{22,26}$ so that decreasing device size alone does not allow one to approach the adiabatic lower limit of energy consumption. We predict electrostatically driven phase change materials to consume less energy and dissipate less heat to the surroundings, therefore offering advantages over thermally driven phase change materials for scaling down device sizes.

In this work, we investigate the theoretical limits of the energy consumption properties of electrostatically driven phase transitions and compare them to thermally driven phase transitions. Taking monolayer $\mathrm{MoTe}_{2}$ and $\mathrm{Ge}_{2} \mathrm{Sb}_{2} \mathrm{Te}_{5}$ alloys as examples, we compute theoretical bounds on energy consumption per unit volume for electrostatically-induced and thermally-induced phase changes, employing density functional theory ${ }^{27,28}$ based approaches to compute the thermal and electrical properties. We find that the electrostatically driven phase transition in monolayer $\mathrm{MoTe}_{2}$ at room temperature has the potential to consume $9 \%$ of the energy per unit volume of the adiabatic lower limit of the thermally driven phase transition in $\mathrm{Ge}_{2} \mathrm{Sb}_{2} \mathrm{Te}_{5}$ alloys. In addition, the experimentally measured energy consumption of a $\mathrm{Ge}_{2} \mathrm{Sb}_{2} \mathrm{Te}_{5}$-based device is several orders of magnitude larger than the adiabatic lower limit, leaving the possibility for monolayer $\mathrm{MoTe}_{2}$ to consume several orders of magnitude less energy than state-of-the-art $\mathrm{Ge}_{2} \mathrm{Sb}_{2} \mathrm{Te}_{5}$-based devices. Furthermore, because monolayer $\mathrm{MoTe}_{2}$ consumes less energy on a pervolume basis than $\mathrm{Ge}_{2} \mathrm{Sb}_{2} \mathrm{Te}_{5}$-based devices, and because $\mathrm{MoTe}_{2}$ devices would be thinner than $\mathrm{Ge}_{2} \mathrm{Sb}_{2} \mathrm{Te}_{5}$-based devices, the savings from reduced volumes has the potential to further reduce the energy required to operate $\mathrm{MoTe}_{2}$-based devices, as well as enable device miniaturization.

Investigations into the kinetics or speed of phase transitions in monolayer $\mathrm{MoTe}_{2}$ and other monolayer materials are ongoing, but much remains unknown about the kinetics of these phase transitions. Experimental studies of thermal phase transitions in $\mathrm{MoTe}_{2}$ have been reported to occur on timescales of several hours at high temperatures, ${ }^{12,14}$ while other reports suggest that the kinetics can occur on timescales of minutes or less at ambient temperatures and below. ${ }^{16,18}$

\section{RESULTS}

Phase diagram of monolayer $\mathrm{MoTe}_{2}$ under electrostatic gating Using the DFT-based approach developed in ref. ${ }^{17}$, we compute the voltage-excess charge phase diagram of monolayer $\mathrm{MoTe}_{2}$ under electrostatic gating. This DFT-based approach models the parallel plate capacitor structure shown in Fig. 1a to gate the monolayer. In this capacitor structure, a dielectric medium of thickness $d$ is sandwiched between the monolayer and a metal plate (i.e., gate electrode). The Mo atoms in the monolayer (colored black in Fig. 1a) are located a distance $s$ away from the dielectric and a gate voltage $V$ is applied to this system. Upon electrostatic gating, excess charge will accumulate in the a

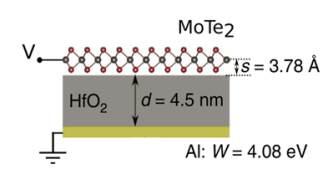

b

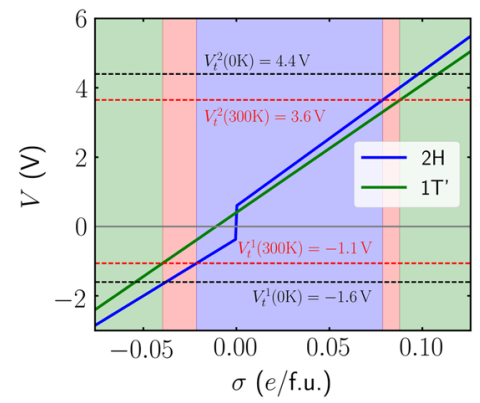

Fig. 1 Structural phase transition in monolayer $\mathrm{MoTe}_{2}$ induced by electrostatic gating. a Illustrates a capacitor structure used to gate the monolayer material to induce a structural phase transition. A dielectric medium of $\mathrm{HfO}_{2}$ of thickness $d=4.5 \mathrm{~nm}$ is sandwiched between the monolayer and a gate electrode, upon which a gate voltage $V$ is applied. The gate electrode is chosen to be aluminum of work function $W=4.08 \mathrm{eV}$. Mo atoms are shown in black, while Te atoms are shown in red. The distance between the Mo atoms in the monolayer and the dielectric is $s=3.78 \AA$. b Computed gate voltage $V$ changing with the excess charge density $\sigma$ in the monolayer. Note that we plot excess charge density instead of excess charge $Q$ on the $x$ axis. Black and red horizontal dashed lines represent the transition voltage at $T=0 \mathrm{~K}$ and $T=300 \mathrm{~K}$, respectively. Note that the transition voltages are lower in magnitude at higher temperatures, as found from a modified form of the Clausius-Clapeyron relation, Eq. (9). Also shown are shaded blue, red, and green regions. The blueshaded region indicates values for which $2 \mathrm{H}-\mathrm{MoTe}_{2}$ is stable at $T=$ $300 \mathrm{~K}$, the red-shaded region indicates that a mixed $2 \mathrm{H}-1 \mathrm{~T}^{\prime}$ phase is most stable at $T=300 \mathrm{~K}$, and the green-shaded region indicates that $1 \mathrm{~T}^{\prime}-\mathrm{MoTe}_{2}$ is most stable at $T=300 \mathrm{~K}$

monolayer, potentially inducing a structural phase transition. Importantly, charge drives the transformation in this process, and not electric field-a monolayer of $\mathrm{MoTe}_{2}$ held at fixed charge as the bias voltage varies will not necessarily undergo the same phase transformation. Because the computational approach used here is described in detail in ref. ${ }^{17}$, we give only a quick review of the method; details on the computational scheme can be found in the reference.

In this approach, the total energy $E(Q)$ of the system when excess charge $Q$ remains fixed on the monolayer consists of three parts:

$E(Q)=E_{\text {mono }}(Q ; s)-Q W+\frac{Q^{2}}{2 C}$.

Here $E_{\text {mono }}$ is the energy of the monolayer, $s$ is a fixed parameter specifying the distance between the dielectric, and the Mo atoms in the monolayer (see Fig. 1a), $-Q W$ is the energy to move charge $Q$ from the gate electrode of work function $W$ to the dielectric, and $Q^{2} / 2 C$ is the energy stored in the dielectric, which has capacitance $C$. The term $E_{\text {mono }}$ in the total energy calculation accounts for Fermi energy changes when electrons are added or removed from the monolayer, so that Eq. (1) does not require an additional term to account for the quantum capacitance. Additional details on the calculation of $E_{\text {mono }}$ are included in the Supplementary Information.

To create the voltage-excess charge phase diagram shown in Fig. $1 \mathrm{~b}$, we find the stationary points of the grand potential $\Phi_{\mathrm{G}}$,

$\Phi_{G}=E(Q)-T S-Q V$,

where $T$ is temperature and $S$ is the total entropy. The stationary points are found at $T=0$, such that

$\left.\frac{\partial \Phi_{\mathrm{G}}(Q, V)}{\partial Q}\right|_{Q=Q_{e q}}=0$. 
The solutions to Eq. (3) provide the points $(Q, V)$ needed to generate a voltage-excess charge phase diagram. Taking the monolayer to be $\mathrm{MoTe}_{2}$, the gate electrode to be aluminum of work function $W=4.08 \mathrm{eV}$, the distance between the Mo atoms and the dielectric to be $s=3.78 \AA$, and the dielectric medium to be $\mathrm{HfO}_{2}$ of thickness $d=4.5 \mathrm{~nm}$ and dielectric constant $k=25$, we compute the gate voltage-excess charge diagram for monolayer $\mathrm{MoTe}_{2}$, as shown in Fig. 1b. Note that in Fig. 1b, the excess charge density $\sigma$ is plotted in e/f.u. instead of total excess charge $Q$. Here, $e$ is the elementary electron charge; positive values of $\sigma$ correspond to the removal of electrons from the monolayer, while negative values correspond to the addition of electrons to the monolayer.

Throughout this work, the monolayer is assumed to be under the mechanical constraint of constant zero stress. This condition is expected to hold when any friction between the monolayer and substrate is small enough that the monolayer can relax freely on the substrate. If friction between the monolayer and substrate is large, a mechanical constraint of constant lattice can be used, where the lattice constants of the material are fixed across the transition. In the fixed lattice case, the phase boundary of $\mathrm{MoTe}_{2}$ will change, so that roughly twice as much charge is required to drive the phase transition, as discussed in ref. ${ }^{17}$. This means that up to four times as much energy will be required to drive the phase transition. This constant lattice case would therefore result in an energy consumption that is roughly $40 \%$ of the adiabatic lower limit of $\mathrm{Ge}_{2} \mathrm{Sb}_{2} \mathrm{Te}_{5}$ energy consumption. However, we again emphasize that this is orders of magnitude lower than the experimentally reported values of $\mathrm{Ge}_{2} \mathrm{Sb}_{2} \mathrm{Te}_{5}$ energy consumption, due to the fact that no waste heat generated by Joule heating is involved in the phase transformation process.

In Fig. 1b, the blue curve represents $2 \mathrm{H}-\mathrm{MoTe}_{2}$, which is discontinuous at $\sigma=0$ due to the band gap of semiconducting $2 \mathrm{H}$ $\mathrm{MoTe}_{2}$, and the green curve represents semimetallic $1 \mathrm{~T}^{\prime}-\mathrm{MoTe}_{2}$. Two zero-temperature transition gate voltages $V_{t}^{1}(T=0 K)=$ $-1.6 \mathrm{~V}$ and $V_{t}^{2}(T=0 \mathrm{~K})=4.4 \mathrm{~V}$ are depicted by the black horizontal dashed lines. Two $T=300 \mathrm{~K}$ transition gate voltages $V_{t}^{1}(T=300 \mathrm{~K})=-1.1 \mathrm{~V}$ and $V_{t}^{2}(T=300 \mathrm{~K})=3.6 \mathrm{~V}$ are depicted by red horizontal dashed lines. The transition voltages at non-zero temperatures are computed using a modified form of the Clausius-Clapeyron relation, Eq. (9). These calculations are described in Section "Impact of non-zero temperature on the phase transition induced by electrostatic gating", as well as the Supplementary Information. An important point is that at nonzero temperatures, the transition voltages become lower in magnitude, therefore reducing the amount of electrostatic energy required to drive a phase transformation.

Because we are interested in applications to phase-change memory devices which operate at roughly room temperature, we indicate in Fig. $1 \mathrm{~b}$ the phase stability properties at $T=300 \mathrm{~K}$. Between the two $T=300 \mathrm{~K}$ transition voltages, semiconducting $2 \mathrm{H}-\mathrm{MoTe}_{2}$ has lower energy and is more stable; this is depicted by blue shading within this region. Outside this range, semimetallic $1 \mathrm{~T}^{\prime}-\mathrm{MoTe}_{2}$ is more thermodynamically favorable; this is depicted by green shading. Between the $2 \mathrm{H}$ and $1 \mathrm{~T}^{\prime}$ stability regions, we predict a coexistence of $2 \mathrm{H}$ and $1 \mathrm{~T}^{\prime}$ in what is labeled a 'Mixed' phase region; this is depicted by red shading in Fig. $1 \mathrm{~b}$. When the gate voltage increases or decreases beyond the positive or negative transition voltage, a transition from $2 \mathrm{H}$ phase to $1 \mathrm{~T}^{\prime}$ phase occurs if done sufficiently slowly for the transformation to occur. Rapid increases in gate voltage could move the material onto the metastable portions of the phase diagram. However, the detailed analysis of the kinetics involved in changing phase between $2 \mathrm{H}$ and $1 \mathrm{~T}^{\prime}$ is beyond this work. We therefore assume thermodynamic equilibrium throughout and a smooth transition from the $2 \mathrm{H}$ to $1 \mathrm{~T}^{\prime}$ phase along a constant voltage path in the mixed-phase region. The nature of the mixed phase of MoTe $\mathrm{M}_{2}$ was recently studied using a phase-field microelasticity model. ${ }^{29}$
In addition, we point out that in general, the transition voltages depend on the dielectric medium used and the dielectric thickness. In particular, the transition voltage for the device in Fig. 1 a has the following dependence on the dielectric thickness $d$,

$V_{t}(d)=\frac{\partial E_{\text {mono }, 2 H}}{\partial Q^{\prime}}\left(Q^{\prime} ; s\right)-W+\frac{Q^{\prime}}{k \epsilon_{0} A} d$,

where $E_{\text {mono, } 2 \mathrm{H}}$ is the energy of the $2 \mathrm{H}$ phase monolayer, $\mathrm{Q}^{\prime}$ is the value of charge at the onset of the mixed phase region, $\epsilon_{0}$ is the permittivity of free space and $A$ is the area of the dielectric in the plane of the monolayer. The transition voltage dependence on the dielectric thickness is described in more detail in ref. ${ }^{17}$.

\section{Comparison of thermally and electrostatically driven phase} transitions

Figure 2 shows a schematic comparison of energy utilization in a thermally driven phase transition of $\mathrm{Ge}_{2} \mathrm{Sb}_{2} \mathrm{Te}_{5}$ and energy utilization in an electrostatically driven phase transition in a monolayer material. Figure $2 \mathrm{a}$, b illustrate temperature changing with entropy in a thermally driven phase transition. In Fig. 2a, crystalline $\mathrm{Ge}_{2} \mathrm{Sb}_{2} \mathrm{Te}_{5}$ alloy is heated up from room temperature to melting temperature and then slowly heated at the melting temperature until the whole alloy is melted. In Fig. 2a, the energy input in this process, $E_{1}$, obtained by integration $\int T \mathrm{~d} S$, is depicted by the gray shaded region. This region is the theoretical lower bound of the energy consumed in driving the phase transformation and is the sum of the energy required to heat up the alloy from room temperature to the melting temperature plus the latent heat at the melting temperature, ${ }^{3}$ equivalent to

$E_{\min }=C\left(T_{\mathrm{m}}-T_{\mathrm{i}}\right)+L$,

where $C$ is the specific heat, $T_{\mathrm{m}}$ is the melting temperature, $T_{\mathrm{i}}$ is the initial temperature which we assume to be room temperature, and $L$ is the latent heat of melting. Using the parameters of standard $\mathrm{Ge}_{2} \mathrm{Sb}_{2} \mathrm{Te}_{5}$ alloy ${ }^{3}\left(T_{\mathrm{m}} \approx 900 \mathrm{~K}, T_{\mathrm{i}}=300 \mathrm{~K}, C=1.25 \mathrm{~J} / \mathrm{K} / \mathrm{cm}^{3}\right.$, and $L=419 \mathrm{~J} / \mathrm{cm}^{3}$, we estimate the energy consumption $E_{1}=$ $E_{\min } \approx 1.17 \mathrm{aJ} / \mathrm{nm}^{3}$, which is the energy input to drive the phase transformation in $\mathrm{Ge}_{2} \mathrm{Sb}_{2} \mathrm{Te}_{5}$ alloy. This quantity is provided in Table 1.

In Fig. $2 b$, the temperature is lowered sufficiently fast that the amorphous $\mathrm{Ge}_{2} \mathrm{Sb}_{2} \mathrm{Te}_{5}$ alloy is quenched to room temperature, as denoted by the arrows and green curve. In the quenching process, an amount of heat, $E_{2}$, is dissipated to the surroundings, depicted by the red shaded region in Fig. $2 b$. This wasted heat is unavoidable in a thermally driven phase transition and it is always nonzero.

Note that the experimentally measured energy input of $\mathrm{Ge}_{2} \mathrm{Sb}_{2} \mathrm{Te}_{5}$ is orders of magnitude larger than the adiabatic lower limit. ${ }^{22}$ The experimentally reported values indicate that the most significant part of the total energy consumption is heat dissipation into the surroundings in the process of Joule heating. The adiabatic lower limit holds only for a thermally isolated device with infinite thermal boundary resistance. Although much research has been conducted to reduce the heat dissipation of a $\mathrm{Ge}_{2} \mathrm{Sb}_{2} \mathrm{Te}_{5}$-based device as much as possible, ${ }^{23-25}$ the experimentally measured energy consumption is still at least 100-10,000 times higher than the adiabatic lower limit. ${ }^{22}$ This ratio increases as the device size scales down.

Because $\mathrm{Ge}_{2} \mathrm{Sb}_{2} \mathrm{Te}_{5}$ alloy has a relatively high melting temperature, we may ask how the energy input to drive a thermal phase transformation in $\mathrm{Ge}_{2} \mathrm{Sb}_{2} \mathrm{Te}_{5}$ compares to the energy input to drive a thermal phase transformation in materials with lower melting temperatures. $\mathrm{VO}_{2}$ has been studied extensively for its metallic to insulating phase transitions in a range of application areas. $^{30,31}$ The commonly studied transitions in vanadates are crystal to crystal rather than the melting transition of $\mathrm{Ge}_{2} \mathrm{Sb}_{2} \mathrm{Te}_{5}$ alloys. The transition temperature of the monoclinic to rutile phase 
Thermally driven phase transition (GST)
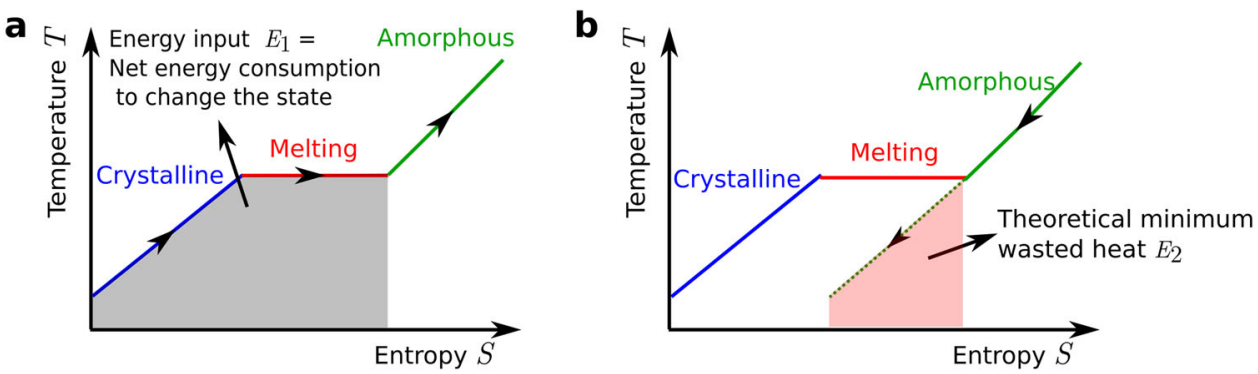

Electrostatically driven phase transition (MoTe2)
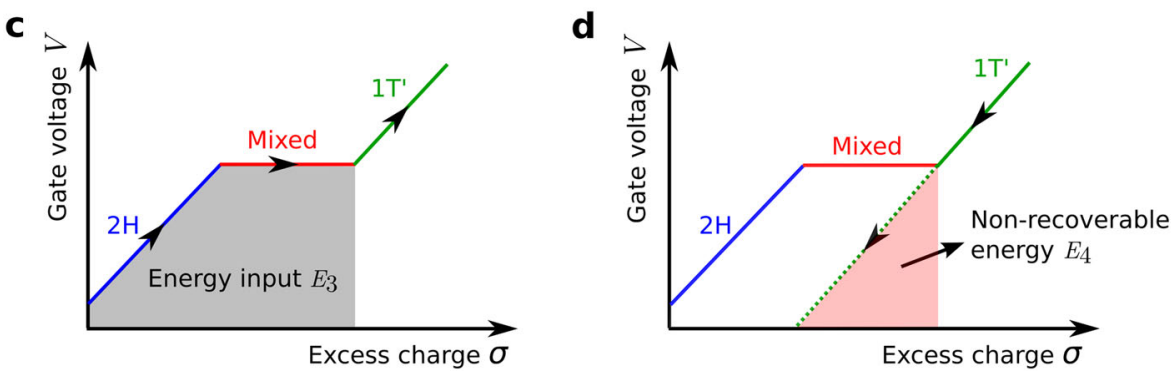

Fig. 2 Comparison of energy consumption in thermally driven phase transitions and electrostatically driven phase transitions. $\mathbf{a}$, $\mathbf{b}$ Illustrations of temperature changing with entropy in a thermally driven phase transition. The phase transition in $\mathrm{Ge}_{2} \mathrm{Sb}_{2} \mathrm{Te}_{5}$ alloy is chosen as an example. To change the state of crystalline $\mathrm{Ge}_{2} \mathrm{Sb}_{2} \mathrm{Te}_{5}$ alloy, it is slowly heated up to the melting temperature and melted to an amorphous phase. In a The gray shaded region represents the energy input to change the state, $E_{1}$. $\mathbf{b}$ The temperature is lowered much faster than the kinetic timescale of the crystallization, the amorphous $\mathrm{Ge}_{2} \mathrm{Sb}_{2} \mathrm{Te}_{5}$ alloy can be quenched into a glassy state, and the red shaded region represents the heat dissipated to the surroundings, $E_{2}$. $\mathbf{c}$, d Illustrate the gate voltage dependence on excess charge in an electrostatically driven phase transition. The phase transition in monolayer $\mathrm{MoTe}_{2}$ is chosen as an example. c The gate voltage on $2 \mathrm{H}_{-}-\mathrm{MoTe}_{2}$ is increased up to the transition voltage, the $2 \mathrm{H}$-to- $1 \mathrm{~T}^{\prime}$ phase transition will occur via a mixed phase thermodynamic region. The gray shaded region represents the energy input in the gating process $E_{3}$ if the voltage or charge are increased much slower than the kinetic timescale of the phase change. $\mathbf{d}$ The gating is rapidly turned off so that $1 \mathrm{~T}^{\prime}-\mathrm{MoTe}_{2}$ will be quenched along the green curve. The red shaded region represents the electrostatic energy that will be lost to the surroundings, $E_{4}$

Table 1. Comparison of energy input to drive a crystalline-toamorphous thermal phase transition in $\mathrm{Ge}_{2} \mathrm{Sb}_{2} \mathrm{Te}_{5}$ alloy, monoclinic to tetragonal thermal phase transition in $\mathrm{VO}_{2}, 2 \mathrm{H}$-to- $1 \mathrm{~T}^{\prime}$ thermal phase transition in $\mathrm{MoTe}_{2}$, and a $2 \mathrm{H}$-to- $1 \mathrm{~T}^{\prime}$ electrostatically driven phase transition in $\mathrm{MoTe}_{2}$ at room temperature

\begin{tabular}{|c|c|c|}
\hline Material & Transtion details & $\begin{array}{l}\text { Energy input } \\
\left(\mathrm{aJ} / \mathrm{nm}^{3}\right)\end{array}$ \\
\hline $\mathrm{VO}_{2}$ & Thermal, adiabatic limit (Eq. (5)) & 0.38 \\
\hline \multirow[t]{2}{*}{$\mathrm{Ge}_{2} \mathrm{Sb}_{2} \mathrm{Te}_{5}$} & Thermal, adiabatic limit (Eq. (5)) & 1.17 \\
\hline & $\begin{array}{l}\text { Joule heating of } 10^{3}-10^{5} \mathrm{~nm}^{3} \\
\text { particles [22] }\end{array}$ & $10^{2}-10^{4}$ \\
\hline \multirow[t]{5}{*}{ Monolayer $\mathrm{MoTe}_{2}$} & Thermal, adiabatic limit (Eq. (5)) & 0.71 \\
\hline & $T=0 \mathrm{~K}$, positive gating & 0.61 \\
\hline & $T=0 \mathrm{~K}$, negative gating & 0.13 \\
\hline & $T=300 \mathrm{~K}$, positive gating & 0.44 \\
\hline & $T=300 \mathrm{~K}$, negative gating & 0.10 \\
\hline \multicolumn{3}{|c|}{$\begin{array}{l}\text { All assume a starting temperature of } T=300 \mathrm{~K} \text { and include the thermal } \\
\text { energy required to heat to the transition temperature plus the latent heat } \\
\text { of the full transition. The } \mathrm{MoTe}_{2} \text { values assume the material is free to slide } \\
\text { on the substrate and the transition occurs at constant zero stress; substrate } \\
\text { friction is expected to increase required energies by up to four times the } \\
\text { values listed. Values are converted to aJ/nm }{ }^{3} \text { using bulk interlayer } \\
\left.\text { distances for } \mathrm{MoTe}_{2} \text {, i.e., } d=6.98 \AA \text { [ } 41\right] \text {. The electrical joule heating } \\
\text { number for } \mathrm{Ge}_{2} \mathrm{Sb}_{2} \mathrm{Te}_{5} \text { is orders of magnitude larger than the adiabatic } \\
\text { limit due to thermal heat flow out of the particle, suggesting that } \\
\text { electrostatically driven mechanism lacking energy flow out of the material } \\
\text { could be far more efficient }\end{array}$} \\
\hline
\end{tabular}

of $\mathrm{VO}_{2}$ is $T_{\mathrm{m}}=340 \mathrm{~K}_{1}^{30}$ well below the transition temperature for $\mathrm{Ge}_{2} \mathrm{Sb}_{2} \mathrm{Te}_{5}$ or bulk MoTe $\mathrm{M}_{2}$. Using (from ref. ${ }^{30}$ ) $T_{i}=300 \mathrm{~K}, \mathrm{C}=3.54 \mathrm{~J} /$ $\mathrm{K} / \mathrm{cm}^{3}$, and $L=241 \mathrm{~J} / \mathrm{cm}^{3}$, we find from Eq. (5) that $E_{\min } \approx 0.38 \mathrm{aJ} /$ $\mathrm{nm}^{3}$ for $\mathrm{VO}_{2}$. This value is also listed in Table 1. Together with the minimum energy input required for a thermal phase transition in $\mathrm{Ge}_{2} \mathrm{Sb}_{2} \mathrm{Te}_{5}$, these values provide a rough estimate of the range of input energies required to drive phase transitions of this type.

While requiring lower energy input to drive a phase transition than $\mathrm{Ge}_{2} \mathrm{Sb}_{2} \mathrm{Te}_{5}$, the low transition temperature of $\mathrm{VO}_{2}$ may preclude its practical use in electrical devices, where temperatures of electrical components may come close to the transition temperature and therefore unintentionally change the phase of the component. For this reason, higher transition temperatures are desired in phase-change memory electronic devices. In addition, $\mathrm{VO}_{2}$ is a volatile material, which precludes its use in non-volatile memory applications.

Figure $2 c, d$ are schematic illustrations of gate voltage changing with excess charge in an electrostatically driven phase transition. For simplicity, we describe here the case of a system at $T=0 \mathrm{~K}$, so that no heat is transferred to the monolayer while the gate voltage is applied. We revisit the case of non-zero temperature in the following section. The curves in Fig. 2c, d are based on the computed $\mathrm{MoTe}_{2}$ phase diagram in Fig. $1 \mathrm{~b}$ and the black dashed $T=0 \mathrm{~K}$ transition voltage line depicted in the figure. When a gate voltage greater than or equal to the transition gate voltage $V_{t}$ (red curve in Fig. 2c) is applied to monolayer $2 \mathrm{H}-\mathrm{MoTe}_{2}$, a $2 \mathrm{H}$-to- $1 \mathrm{~T}^{\prime}$ phase transition will occur in the monolayer. At the transition gate voltage, the monolayer is a mixed phase of $2 \mathrm{H}$ and $1 \mathrm{~T}^{\prime}$, analogous to the melting process shown in Fig. $2 \mathrm{a}$. As the region of $1 \mathrm{~T}^{\prime}$ phase grows in the mixed phase, the excess charge $Q$ in the monolayer 
a

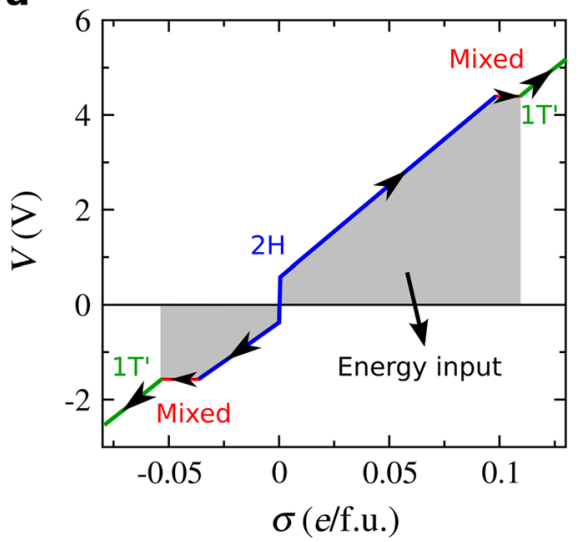

b

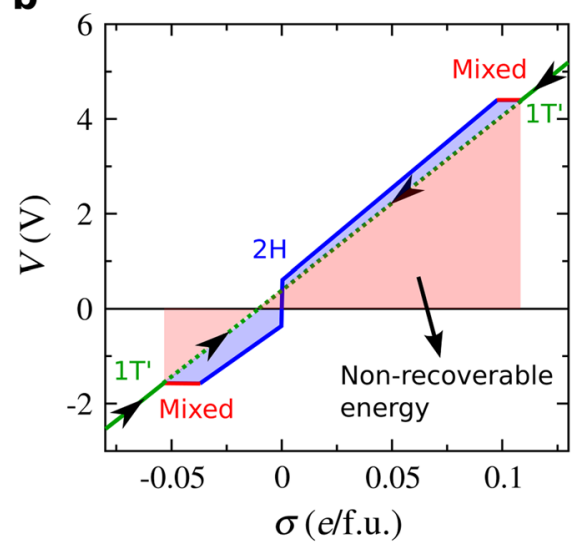

Fig. 3 Phase transition path in monolayer $\mathrm{MoTe}_{2}$ under electrostatic gating. $\mathbf{a}, \mathbf{b}$ These figures show the phase transition path in monolayer $\mathrm{MoTe}_{2}$ under electrostatic gating, as schematically illustrated in Fig. 2c, d. In each figure, there are two sets of arrows, representing the phase change under both positive charging and negative charging. The meanings of the shaded regions are the same as in Fig. $2 \mathrm{c}$, $\mathrm{d}$

will increase while the gate voltage is maintained at the transition voltage. The gray shaded region shown in Fig. 2c represents the minimum energy input (i.e., $E_{3}$ ) needed to transform monolayer $\mathrm{MoTe}_{2}$ from the $2 \mathrm{H}$ phase to the $1 \mathrm{~T}^{\prime}$ phase. This quantity assumes that the voltage is increased sufficiently slowly, so that the system remains at thermodynamic equilibrium throughout. Physically, this means that the voltage increases on a timescale much slower than the kinetics of the $2 \mathrm{H}$ to $1 \mathrm{~T}^{\prime}$ transition.

Figure $2 \mathrm{~d}$ shows that upon reducing the voltage sufficiently fast, the material remains in the $1 \mathrm{~T}^{\prime}$ phase and excess charge flows out of the monolayer, denoted by the arrows and the green curve. In this plot, it is assumed that the voltage is reduced on a timescale faster than the kinetics of the $1 \mathrm{~T}^{\prime}$ to $2 \mathrm{H}$ transition, so that the material remains metastable in the $1 \mathrm{~T}^{\prime}$ state in analog to the quenching process in Fig. $2 \mathrm{~b}$.

Importantly, the two cases of thermally driven and electrostatically driven phase transitions differ in a fundamental way. Figure $2 \mathrm{~d}$ shows that, in principle, an amount of electrostatic energy, $E_{4}$, has the potential to be recovered by the system. The gated 2D material system is essentially a charged capacitor. This energy could potentially be recovered by discharging the capacitor into a reservoir or external capacitor. In the limit that this energy can be recovered with perfect efficiency, the net energy consumption to drive a phase transition from $2 \mathrm{H}$ to $1 \mathrm{~T}^{\prime}$ and subsequently relax to $2 \mathrm{H}$ is $E_{3}-E_{4}$. However, electronic devices are not typically configured to recover such energy and we therefore assume this energy to be lost to the surrounding environment.

Given the qualitative picture of the phase transformations in monolayer $\mathrm{MoTe}_{2}$ and $\mathrm{Ge}_{2} \mathrm{Sb}_{2} \mathrm{Te}_{5}$ in Fig. 2, we now compute the zero-temperature energy input required to drive the phase transition in monolayer $\mathrm{MoTe}_{2}$. Figure $3 \mathrm{a}$ shows the $T=0 \mathrm{~K}$ transition process of monolayer $\mathrm{MoTe}_{2}$ under electrostatic gating for the device specifications of Fig. 1a. The gray shaded region represents the input energy required to drive the $2 \mathrm{H}$-to- $1 \mathrm{~T}^{\prime}$ phase transition, analogous to the transition path in Fig. 2c. Figure $3 \mathrm{~b}$ shows the transition path when the gate voltage is turned off, analogous to Fig. $2 d$. The red shaded region in Fig. $3 \mathrm{~b}$ represents the electrostatic energy which we assume to be lost. The energy input to drive the phase transition is

$E(T=0 K)=\int_{2 H} V d Q+\int_{\text {mixed }} V d Q$,

where the first integral is taken over the blue $2 \mathrm{H}$ transition path in Fig. $3 a$ and the second integral is taken over the red mixed

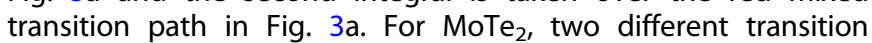

pathways can be used: one for positive charging and the other for negative charging.

From Fig. $3 \mathrm{a}$ at $T=0 \mathrm{~K}$, we find that the energy input per volume for Eq. (6) at positive bias is $0.61 \mathrm{aJ} / \mathrm{nm}^{3}$, while the energy input for negative bias is $0.13 \mathrm{aJ} / \mathrm{nm}^{3}$. These values are also listed in Table 1 and compared to the $T=300 \mathrm{~K}$ values. Note that to get a pervolume comparison of monolayer $\mathrm{MoTe}_{2}$, we use the bulk $2 \mathrm{H}$ $\mathrm{MoTe}_{2}$ interlayer distance $d=6.98 \AA$ to compute a volume per formula unit of $\mathrm{MoTe}_{2}$.

Impact of non-zero temperature on the phase transition induced by electrostatic gating

The phase transition diagram in Fig. 3 is drawn at zero temperature. To model a practical phase change memory device, we would like to know how the device in Fig. 1a would operate at room temperature. The effects of non-zero temperature on the device are important in two different respects. First, heat transfer between the monolayer and the surrounding environment can occur during the phase transformation, adding an important term to the total energy consumption. This can be seen via reference to the total differential of internal energy, which at non-zero temperature and constant zero stress becomes

$d E=T d S+V d Q$

Second, it has been shown that non-zero temperatures can have a large quantitative impact on the voltage-temperature phase boundary. ${ }^{11,15}$ The reported $2 \mathrm{H}$-to- $1 \mathrm{~T}^{\prime}$ phase transition at high temperature $^{10}$ suggests that the transition voltage should decrease as temperature increases, and our results corroborate this finding. This is important because it indicates that at higher temperatures, less electrostatic energy is needed to drive the phase transformation. In this section, we discuss both the heat transferred to the monolayer during the phase transformation and the temperature dependence of the gate voltage using a modified form of the Clausius-Clapeyron relation. Details on the calculation are included in the Supplementary Information.

We first consider the amount of heat transferred to the monolayer under an applied voltage at non-zero temperature. As a thermodynamic constraint, we consider an isothermal process in which the monolayer and all other components of the system shown in Fig. 1a remain at a constant temperature throughout the phase transformation of the monolayer. This constraint applies for cases in which phase-change memory devices operate at roughly room temperatures and heat is allowed to flow in and out of the device. Theoretically, adiabatic 
a

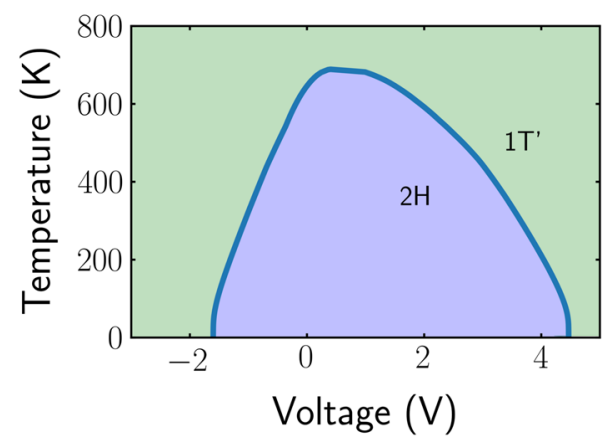

b

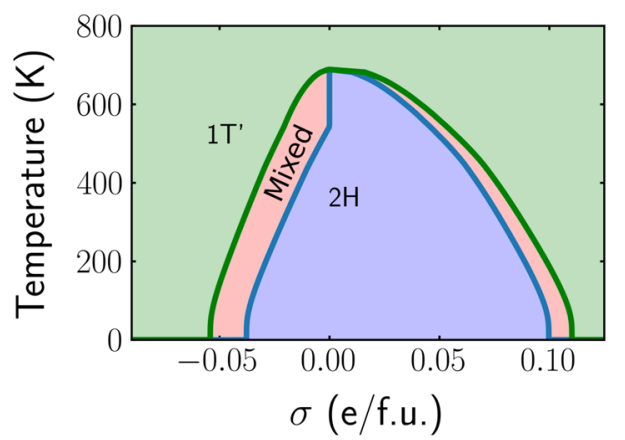

Fig. 4 Temperature-voltage and temperature-excess charge phase diagrams for the device in Fig. 1a, computed using the modified Clausius-Clapeyron relation in Eq. 9. a Temperature-gate voltage phase diagram of monolayer MoTe $\mathrm{M}_{2}$ under electrostatic gating. In the blue shaded region, semiconducting $2 \mathrm{H}$ phase is more stable; in the green shaded region, semi-metallic $1 \mathrm{~T}^{\prime}$ phase is more stable. $\mathbf{b}$ Temperatureexcess charge phase diagram of monolayer $\mathrm{MoTe}_{2}$ under electrostatic gating. In addition to blue and green shading, the red shading indicates regions where a mixed $2 \mathrm{H}$ and $1 \mathrm{~T}^{\prime}$ phase is most stable. The peak value of temperature is $T=690 \mathrm{~K}$. Note that for conditions of constant lattice, rather than constant zero stress, the amount of excess charge required to drive a transition will be approximately twice as large as the values shown here, as discussed in ref. ${ }^{17}$

constraints are also possible, but we do not consider such constraints in this work.

When the system is held at constant temperature, the main contribution to the heat flow into the system comes from the change in entropy in transforming from the $2 \mathrm{H}$ to $1 \mathrm{~T}^{\prime}$ phase, $\Delta S=$ $S_{1 T^{\prime}}-S_{2 H}$. Given this entropy change, we can reimagine the phase transformation process of Fig. 3 via reference to Fig. 1b. As in Fig. 3, we may consider a process in which the monolayer starts in the $2 \mathrm{H}$ phase at $V=0 \mathrm{~V}$ and a fixed temperature $T=300 \mathrm{~K}$. Upon gating the device, charge enters the monolayer and for voltages of sufficiently small magnitude, the monolayer remains in the $2 \mathrm{H}$ phase, exactly as depicted in the blue path of Fig. 3a. For all points along this blue path, any entropy change during this process is regarded as negligible, since no phase transformation has occurred.

Because the device now operates at room temperature, the magnitude of gating voltage necessary for $\mathrm{MoTe}_{2}$ to enter a mixed phase is smaller than in the $T=0 \mathrm{~K}$ case (see Fig. 4a). The monolayer must be gated to $V_{t}^{1}(T=300 \mathrm{~K})$ in the negative bias case or $V_{t}^{2}(T=300 \mathrm{~K})$ in the forward bias case for the phase transformation to occur (see Fig. 1b). When reaching the $T=300 \mathrm{~K}$ transition voltage, the bias is held fixed and the system enters a mixed phase of $2 \mathrm{H}$ and $1 \mathrm{~T}^{\prime}$. Upon completing the transformation from $2 \mathrm{H}$ to $1 \mathrm{~T}^{\prime}$, the monolayer has experienced an entropy change of $\Delta S(T=300 \mathrm{~K})$, and therefore an additional latent heat energy $L$ $=T \Delta S$ is required to drive the transformation.

Upon transforming to the $1 \mathrm{~T}^{\prime}$ phase, the gate voltage is decreased quickly so that the monolayer remains metastable in the $1 \mathrm{~T}^{\prime}$ phase, exactly as is shown in the green dashed line of Fig. $3 \mathrm{~b}$. As in the zero-temperature case, we assume any electrostatic energy to be lost to the surroundings during this process, so that no energy is recovered in this process.

To compute the entropy change $\Delta S$, we note that the total entropy of the $2 \mathrm{H}$ or $1 \mathrm{~T}^{\prime}$ phase is the sum of a vibrational (phonon) contribution and an electronic contribution, so that $\triangle S(T$, $\left.Q_{2 \mathrm{H}}, Q_{1 \mathrm{~T}^{\prime}}\right)=\Delta S^{\mathrm{ph}}(T)+\Delta S^{\mathrm{el}}\left(T, Q_{2 \mathrm{H}^{\prime}}, Q_{1 \mathrm{~T}^{\prime}}\right)$. Note that the electronic entropy difference $\Delta S^{\mathrm{el}}$ depends on both temperature $T$ and the excess charge of the $2 \mathrm{H}$ and $1 \mathrm{~T}^{\prime}$ phases, $Q_{2 \mathrm{H}}$ and $Q_{1 \mathrm{~T}^{\prime}}$. This is due to the fact that adding charge changes the Fermi level of each phase, altering the local density of states that gives rise to the occupational entropy. Technically, the phonon contribution to the entropy difference $\Delta S^{\text {ph }}$ can also depend on the excess charge of each phase, but these effects are small and are therefore ignored below, as discussed in the Supplementary Information.

We compute the phonon contribution to the entropy using the phonon density of states at zero excess charge $(Q=0)$ using VASP and the Phonopy package ${ }^{32}$ (see the Supplementary Information for details). The electronic contribution is computed using VASP with electronic Fermi smearing to determine the entropy from partial electronic occupancies of the Kohn-Sham states. We compute the electronic entropy for both the $2 \mathrm{H}$ and $1 \mathrm{~T}^{\prime}$ phases at a large range of values of excess charge and temperature, as shown in Supplementary Information Figure 2.

Because the electronic entropy difference between the $2 \mathrm{H}$ and $1 \mathrm{~T}^{\prime}$ phases depends on the sign of charge added to the monolayer at a given temperature, the latent heat term associated with the transformation also depends on the sign of charge added to the monolayer. The exact values of the electronic entropy difference between the $2 \mathrm{H}$ and $1 \mathrm{~T}^{\prime}$ phases along the phase transformation boundary are shown in Supplementary Information Figure 4. The values in this figure can be used to compute the latent heat at all temperatures up to the zero excess charge transition temperature.

Given the computed change in entropy $\Delta S^{\text {ph }}$ between the $2 \mathrm{H}$ and $1 \mathrm{~T}^{\prime}$ phases from the phonon density of states at $T=300 \mathrm{~K}$ and the electronic difference in entropy $\Delta S^{\mathrm{el}}$ at $T=300 \mathrm{~K}$ and the corresponding values of $Q_{2 \mathrm{H}}$ and $Q_{1 \mathrm{~T}^{\prime}}$ shown in Fig. $1 \mathrm{~b}$ for both positive and negative values of $V$, we compute the latent heat transferred to the system as

$$
L=T\left(\Delta S^{p h}+\Delta S^{e l}\right)= \begin{cases}0.023 a J / n m^{3} & (V>0) \\ 0.029 a J / n m^{3} & (V<0)\end{cases}
$$

This quantity is roughly $5 \%$ of the total energy input required to drive a phase transformation at $T=300 \mathrm{~K}$ for the forward bias $(V>$ 0 ) case and roughly $30 \%$ of the total energy input for the reverse bias $(V<0)$ case.

Although the latent heat $L$ adds to the total energy required to drive the $2 \mathrm{H}$ to $1 \mathrm{~T}^{\prime}$ phase transformation, it turns out that the amount of electrostatic energy required to drive the phase transformation at $T=300 \mathrm{~K}$ actually decreases significantly from the $T=0 \mathrm{~K}$ case. Using a modified form of the Clausius-Clapeyron relation,

$\frac{d V}{d T}=-\frac{\Delta S}{\Delta Q}$,

where $\Delta Q=Q_{1 T^{\prime}}(V)-Q_{2 H}(V)$, we compute the temperature-voltage phase diagram shown in Fig. 4a (see the Supplementary Information for details). By combining this temperature-voltage phase diagram with the voltage-excess charge data in Fig. $1 \mathrm{~b}$, we compute the temperature-excess charge phase diagram shown in Fig. 4b. The phase diagrams in Fig. 4 indicate that the magnitudes of the voltage and/or excess charge along the phase boundaries decrease as temperature is increased. The semiconducting $2 \mathrm{H}$ phase is more 
stable in the blue shaded region, the semimetallic $1 \mathrm{~T}^{\prime}$ phase is more stable in the green shaded region, and a mixed phase is most stable in the red shaded region. The transition gate voltage increases as temperature decreases and achieves its maximum at $T=0 \mathrm{~K}$. We also find that at $Q=0$, the transition temperature is roughly $T=690 \mathrm{~K}$. This agrees well with ref. ${ }^{11}$, which predicts that under stress free conditions, the single layer $2 \mathrm{H}$ phase will transform to the $1 \mathrm{~T}^{\prime}$ phase at $T=610 \mathrm{~K}$ without electrostatic gating.

The total energy input to drive a phase transition in monolayer $\mathrm{MoTe}_{2}$ is the sum of the electrostatic energy input and latent heat (Eq. 8) at room temperature. For the electrostatic energy input, we integrate $\int V d Q$ along the $2 \mathrm{H}$ transition path up to the transition voltage $V_{t}^{T}, 2(T=300 K)$, so that the total energy consumption at $T$ $=300 \mathrm{~K}$ becomes

$E(T=300 \mathrm{~K})=\int \underset{\substack{2 \mathrm{H} \\ 300 \mathrm{~K}}}{ } V \mathrm{~d} Q+\iint_{\substack{\text { mixed } \\ 300 \mathrm{~K}}} V \mathrm{~d} Q+L$.

The $T=300 \mathrm{~K}$ energy input for forward and reverse bias using Eq. (10) are 0.44 and $0.10 \mathrm{aJ} / \mathrm{nm}^{3}$, respectively. For comparison, the $T$ $=0 \mathrm{~K}$ electrostatic energy input values are 0.61 and $0.13 \mathrm{aJ} / \mathrm{nm}^{3}$. These values are listed in Table 1.

As a final point of interest, we also compute the energy input required to drive a thermal $2 \mathrm{H}$ to $1 \mathrm{~T}^{\prime}$ phase transition in monolayer $\mathrm{MoTe}_{2}$. We consider heating the monolayer at zero excess charge from $T=300 \mathrm{~K}$ up to the computed transition temperature $T=690 \mathrm{~K}$ found in Fig. 4. This value is exactly analogous to the adiabatic energy input required to drive the phase transition of $\mathrm{Ge}_{2} \mathrm{Sb}_{2} \mathrm{Te}_{5}$ alloy and $\mathrm{VO}_{2}$ in Eq. (5). In Supplementary Information Figure 5 , we compute the heat capacity of monolayer $2 \mathrm{H}-\mathrm{MoTe}_{2}$ to be $C_{2 \mathrm{H}}=1.61 \mathrm{~J} / \mathrm{K} / \mathrm{cm}^{3}$ at $T=$ $690 \mathrm{~K}$. This is very close to experimentally reported values of bulk $2 \mathrm{H}$-phase $\mathrm{MoTe}_{2}, \mathrm{C}=1.65 \mathrm{~J} / \mathrm{K} / \mathrm{cm}^{3}{ }^{33,34}$ In addition, we use Supplementary Information Figure 3 to compute $\Delta S^{\text {ph }}+\Delta S^{\mathrm{el}}$ at $T$ $=690 \mathrm{~K}$. Using these values, we find $C_{2 \mathrm{H}}\left(T_{\mathrm{m}}-T_{\mathrm{i}}\right) \approx 0.63 \mathrm{aJ} / \mathrm{nm}^{3}$ and $L=0.08 \mathrm{aJ} / \mathrm{nm}^{3}$, leading to (from Eq. 5) $E_{\min }=0.71 \mathrm{aJ} / \mathrm{nm}^{3}$. This value is also provided in Table 1. For comparison, note that Song et al. $^{16}$ report the latent heat of Te-deficient $\mathrm{MoTe}_{2}$ powder to be $L=0.03 \mathrm{aJ} / \mathrm{nm}^{3}$ for measurements done at $T=1128 \mathrm{~K}$.

We also point out that recent experimental investigations report that the thermal boundary resistance (TBR) of $\mathrm{MoS}_{2}$ on a $\mathrm{SiO}_{2}$ substrate is around $70 \mathrm{~m}^{2} / \mathrm{GW} / \mathrm{K}_{1}^{35}$ while the TBR of $\mathrm{Ge}_{2} \mathrm{Sb}_{2} \mathrm{Te}_{5}$ on a $\mathrm{SiO}_{2}$ substrate is around $30 \mathrm{~m}^{2} / \mathrm{GW} / \mathrm{K}^{36}$ For $\mathrm{MoTe}_{2}$, the TBR is expected to be even higher than that of $\mathrm{MoS}_{2}$ because the larger mass density per unit area of $\mathrm{MoTe}_{2}$ gives rise to higher acoustic impedance. These findings indicate that thermally driven monolayer TMD-based phase change memory devices should naturally dissipate less heat to the surroundings than $\mathrm{Ge}_{2} \mathrm{Se}_{2} \mathrm{Te}_{5}$-based devices. However, we again emphasize that the electrostatically driven phase change mechanism in monolayer $\mathrm{MoTe}_{2}$ is distinct from this thermal mechanism, and that in the electrostatically driven case, heat dissipation to the surroundings is a relatively small contribution to the total per-volume energy consumption.

\section{DISCUSSION}

Table 1 shows that the $T=300 \mathrm{~K}$ electrostatically driven phase transformation of monolayer $\mathrm{MoTe}_{2}$ from the $2 \mathrm{H}$ to $1 \mathrm{~T}^{\prime}$ phase under constant zero stress conditions requires roughly 38 and $9 \%$ of the energy input per unit volume of the adiabatic lower limit to drive a phase transition in $\mathrm{Ge}_{2} \mathrm{Sb}_{2} \mathrm{Te}_{5}$ for positive and negative voltage bias, respectively. Furthermore, the adiabatic lower limit $\mathrm{Ge}_{2} \mathrm{Sb}_{2} \mathrm{Te}_{5}$ energy consumption is $100-10,000$ times smaller than the experimentally measured values due primarily to waste heat generated in the process of Joule heating of $\mathrm{Ge}_{2} \mathrm{Sb}_{2} \mathrm{Te}_{5}$-based devices. This opens the opportunity for monolayer $\mathrm{MoTe}_{2}$ to consume several orders of magnitude less energy per unit volume than $\mathrm{Ge}_{2} \mathrm{Sb}_{2} \mathrm{Te}_{5}$.
Fabricated devices that employ monolayer or few layer forms of $\mathrm{MoTe}_{2}$, rather than bulk, may see an additional energy savings from smaller thicknesses leading to smaller overall volumes. Chemical stability at very thin thicknesses may be a potential advantage of using layered materials over bulk materials. However, tunneling current through the device is expected to place a practical limit on the minimum thickness of the material. For devices that can be made from a single or few layer thickneses with acceptable performance, additional energy savings are expected that are not explicitly accounted for in our energy per unit volume numbers given in Table 1 . We also point out that the write energy per bit for a typical $\mathrm{Ge}_{2} \mathrm{Sb}_{2} \mathrm{Te}_{5}$ phase change device is around $10 \mathrm{pJ}$ for cells roughly of size $10 \times 10 \times 10 \mathrm{~nm}^{3}, 6,23$ including thermal conduction effects. For a relatively large $50 \times$ $50 \times 0.5 \mathrm{~nm}^{3}$ monolayer $\mathrm{MoTe}_{2}$ storage bit, our calculations predict the write energy per bit is on the order of $0.1 \mathrm{fJ}$, roughly five orders of magnitude smaller than $\mathrm{Ge}_{2} \mathrm{Sb}_{2} \mathrm{Te}_{5}$ devices.

The calculations of critical charge required to drive the transition in ref. ${ }^{17}$ indicate that the nature of the mechanical constraint of the material has a large impact on the critical charge required. The difference in charge required differs by approximately a factor of two between the constant zero stress case (shown in Table 1) and the fixed lattice case. In the former, the monolayer is assumed to be able to slide freely on the substrate, while the latter case assumes substrate friction sufficient to constrain the lattice constants. A recent electrostatic gating experimental report indicates that the transition is centered around a charge density associated with the fixed lattice case of ref. ${ }^{17}$, suggesting that the appropriate mechanical constraint may be closer to the fixed lattice case for a monolayer on a substrate. The energy consumption for the fixed lattice case is expected to be roughly four times larger than the constant zero stress case, due to larger critical transition charges. However, this quantity is still orders of magnitude lower than the experimentally reported $\mathrm{Ge}_{2} \mathrm{Sb}_{2} \mathrm{Te}_{5}$ energy consumption values in Table 1.

There may exist opportunities for engineering single layer materials to consume less energy than those shown in Table 1. For example, ref. ${ }^{17}$ discusses the potential for the alloy $\mathrm{Mo}_{x} \mathrm{~W}_{1-x} \mathrm{Te}_{2}$ to exhibit the transition at smaller voltages and charge densities, tunable with alloy composition $x$. Moving the transition voltage closer to zero is expected to reduce the energy requirements in Table 1. Figure 4a shows a similar reduction in transition voltage can be achieved with increasing operating temperature. The calculations of ref. ${ }^{11}$ and ref. ${ }^{15}$ report that tensile strain moves the transition closer to ambient conditions, and may constitute a further mechanism for reduction of the critical gate voltages and and energy consumption.

Finally, the kinetics of this transition are expected to play a critical role in the utility of practical devices. As previously discussed, investigations into the kinetics of phase transitions in monolayer $\mathrm{MoTe}_{2}$ and other monolayer materials are nascent and much remains unknown. Experimental studies of thermal phase transitions in $\mathrm{MoTe}_{2}$ have been reported to occur on timescales of several hours at high temperatures, ${ }^{12,14}$ while other reports suggest that the kinetics can occur on timescales of minutes or less at ambient temperatures and below. ${ }^{16,18}$ The large variability in these reports suggests that there is potential to engineer the kinetics of these phase transitions to achieve desired electronic or other device timescales.

In this work, we compared the energy consumption in electrostatically driven phase transitions of monolayer $\mathrm{MoTe}_{2}$ to the energy consumption in thermally driven phase transitions of $\mathrm{Ge}_{2} \mathrm{Sb}_{2} \mathrm{Te}_{5}$ alloys. In the thermally driven phase transition of $\mathrm{Ge}_{2} \mathrm{Sb}_{2} \mathrm{Te}_{5}$, the vast majority of energy consumed is dissipated as waste heat to the surrounding environment, thus limiting energy efficiency and miniaturization of device sizes. In electrostatically driven phase transitions of monolayer $\mathrm{MoTe}_{2}$, however, we find that only a small amount of heat enters and leaves the system, so 
that heat dissipation is not as great an issue for device miniaturization. We discover that the net energy consumption per unit volume in $\mathrm{MoTe}_{2}$ is $9 \%$ of the adiabatic lower limit of energy consumption per unit volume in $\mathrm{Ge}_{2} \mathrm{Sb}_{2} \mathrm{Te}_{5}$ alloy. Furthermore, the adiabatic lower limit of energy consumption in $\mathrm{Ge}_{2} \mathrm{Sb}_{2} \mathrm{Te}_{5}$ alloy is several orders of magnitude lower than experimentally-measured values. These factors may enable electrostatic devices based on monolayer $\mathrm{MoTe}_{2}$ and potentially other 2D-materials to consume several orders of magnitude less energy than their $\mathrm{Ge}_{2} \mathrm{Sb}_{2} \mathrm{Te}_{5}$ counter-parts.

\section{METHODS}

All periodic DFT calculations were performed with the Vienna Ab initio Simulation Package (VASP), ${ }^{37}$ version 5.3.3 and/or 5.3.5. The calculations use the projector augmented-wave ${ }^{38}$ method and the electron exchangecorrelation interaction was treated by the generalized gradient approximation (GGA) functional of Perdew, Burke, and Ernzerhof (PBE). ${ }^{39}$ A kinetic energy cutoff of $350 \mathrm{eV}$ for the plane-wave basis set was used for all calculations. All atomic structures and in-plane lattice constants were relaxed using the conjugate gradient algorithm. The convergence thresholds for ionic and electronic relaxations were $0.5 \times 10^{-7} \mathrm{eV}$ per formula unit and $0.5 \times 10^{-8} \mathrm{eV}$ per formula unit, respectively. A 2 f.u. unit cell with an $18 \times 18 \times 1$ Monkhorst-Pack ${ }^{40} \mathrm{k}$-point mesh for the Brillouin zone sampling was employed for the calculations done in Figs. 1 and 3. These Figures also used a $36 \AA$ vacuum space along the $z$ direction (perpendicular to the plane of the monolayer) to prevent interaction between periodic cell repeats, as well as a Gaussian electronic energy distribution smearing of $50 \mathrm{meV}$ to aid in energy convergence. These calculations also included non-colinear spinorbit coupling. The electronic entropies of $2 \mathrm{H}$ and $1 \mathrm{~T}^{\prime}$ phases of $\mathrm{MoTe}_{2}$ were computed directly from VASP using Fermi-Dirac smearing without spin-orbit coupling (details are found in the Supplementary Information). The vibrational (phonon) entropies of $2 \mathrm{H}$ - and $1 \mathrm{~T}^{\prime}-\mathrm{MoTe}_{2}$ were computed from the phonon density of states using the Phonopy ${ }^{32}$ Python package (see the Supplementary Information for details). These phonon calculations used VASP's built-in density functional perturbation theory method (IBRION $=8$ ) and a $2 \times 2 \times 1$ ( 8 f.u.) supercell generated from the relaxed lattice positions of the $2 \mathrm{f}$.u. unit cell used in Figs. 1 and 3. The supercell uses a vacuum space of $16 \AA$ along the $z$ direction and an $18 \times 18 \times 1$ MonkhorstPack k-point mesh. These simulations also included non-colinear spin-orbit coupling, with an electronic relaxation threshold of $0.5 \times 10^{-5} \mathrm{eV} / \mathrm{f}$.u.

\section{Data availability}

All code and data used to generate figures and results in this paper can be found at https://github.com/rehnd/MoTe2PhaseChangeEnergy.

\section{ACKNOWLEDGEMENTS}

This work was partially supported by NSF grants EECS-1436626 and DMR-1455050, Army Research Office grant W911NF-15-1-0570, Office of Naval Research grant N00014-15-1-2697, and a seed grant from Stanford System X Alliance. This work was supported in part by the U. S. Army Research Laboratory, through the Army High Performance Computing Research Center, Cooperative Agreement W91 1NF-07-0027.

\section{AUTHOR CONTRIBUTIONS}

D.A.R. performed simulations of the thermal properties of monolayer $\mathrm{MoTe}_{2}$ and prepared the manuscript. Y.L. performed simulations of the zero temperature voltage-excess charge phase diagram. E.P. provided insights into the use of thermally-driven phase change materials and revised the manuscript. Y.L., D.A.R., and E.J.R. collectively worked on the interpretation of data and simulation design. E.J. R. served as the primary advisor for the project.

\section{ADDITIONAL INFORMATION}

Supplementary information accompanies the paper on the npj Computational Materials website (https://doi.org/10.1038/s41524-017-0059-2).

Competing interests: The authors declare no competing financial interests.

Publisher's note: Springer Nature remains neutral with regard to jurisdictional claims in published maps and institutional affiliations.

\section{REFERENCES}

1. Zalba, B., Marín, J. M., Cabeza, L. F. \& Mehling, H. Review on thermal energy storage with phase change: materials, heat transfer analysis and applications. Appl. Therm. Eng. 23, 251-283 (2003).

2. Hudgens, S. \& Johnson, B. Overview of phase-change chalcogenide nonvolatile memory technology. MRS Bull. 29, 829-832 (2004).

3. Raoux, S., Xiong, F., Wuttig, M. \& Pop, E. Phase change materials and phase change memory. MRS Bull. 39, 703-710 (2014).

4. Lee, B.-S. et al. Investigation of the optical and electronic properties of $\mathrm{Ge}_{2} \mathrm{Sb}_{2} \mathrm{Te}_{5}$ phase change material in its amorphous, cubic, and hexagonal phases. J. Appl. Phys. 97, 093509 (2005).

5. Wuttig, M. \& Yamada, N. Phase-change materials for rewriteable data storage. Nat. Mater. 6, 824 (2007).

6. Yu, S. \& Chen, P.-Y. Emerging memory technologies: recent trends and prospects. IEEE Solid-State Circuits Mag. 8, 43-56 (2017).

7. Meena, J., Sze, S., Chand, U. \& Tseng, T.-Y. Overview of emerging nonvolatile memory technologies. Nanoscale Res. Lett. 9, 526 (2014).

8. Sandoval, S. J., Yang, D., Frindt, R. \& Irwin, J. Raman study and lattice dynamics of single molecular layers of MoS $_{2}$. Phys. Rev. B 44, 3955 (1991).

9. Zhou, Y. \& Reed, E. J. Structural phase stability control of monolayer $\mathrm{MoTe}_{2}$ with adsorbed atoms and molecules. J. Phys. Chem. C. 119, 21674-21680 (2015).

10. Brown, B. E. The crystal structures of $\mathrm{WTe}_{2}$ and high-temperature $\mathrm{MoTe}_{2}$. Acta Crystallogr. 20, 268-274 (1966).

11. Duerloo, K.-A. N. \& Reed, E. J. Structural phase transitions by design in monolayer alloys. ACS Nano 10, 289-297 (2015).

12. Oliver, S. M. et al. The structural phases and vibrational properties of $\mathrm{Mo}_{1-x} \mathrm{~W}_{x} \mathrm{Te}_{2}$ alloys. 2D Mater. 4, 045008 (2017).

13. Rhodes, D. et al. Engineering the structural and electronic phases of $\mathrm{MoTe}_{2}$ through w substitution. Nano Lett. 17, 1616-1622 (2017).

14. Empante, T. A. et al. Chemical vapor deposition growth of few-layer $\mathrm{MoTe}_{2}$ in the $2 \mathrm{H}, 1 \mathrm{~T}$, and $1 \mathrm{~T}$ phases: tunable properties of $\mathrm{MoTe}_{2}$ films. ACS Nano 11, 900-905 (2017).

15. DuerlooK. -A. N., LiY.. \& ReedE. J.. Structural phase transitions in two-dimensional mo-and w-dichalcogenide monolayers. Nat. Commun. 5, 4214 (2014).

16. Song, S. et al. Room temperature semiconductor-metal transition of $\mathrm{MoTe}_{2}$ thin films engineered by strain. Nano Lett. 16, 188-193 (2015).

17. Li, Y., Duerloo, K.-A. N., Wauson, K. \& Reed, E. J. Structural semiconductor-tosemimetal phase transition in two-dimensional materials induced by electrostatic gating. Nat. Commun. 7, 10671 (2016).

18. Wang, Y. et al. Structural phase transition in monolayer $\mathrm{MoTe}_{2}$ driven by electrostatic doping. Nature 550, 487-491 (2017).

19. Tsen, A. W. et al. Structure and control of charge density waves in twodimensional 1T-TaS, Proc. Natl. Acad. Sci. 112, 15054-15059 (2015).

20. Yoshida, M., Suzuki, R., Zhang, Y., Nakano, M. \& Iwasa, Y. Memristive phase switching in two-dimensional 1T-TaS 2 crystals. Sci. Adv. 1, e1500606 (2015).

21. Yoshida, M. et al. Controlling charge-density-wave states in nano-thick crystals of 1T-TaS 2 . Sci. Rep. 4, 7302 (2014).

22. Lacaita, A. L. \& Redaelli, A. The race of phase change memories to nanoscale storage and applications. Microelectron. Eng. 109, 351-356 (2013).

23. Xiong, F., Liao, A. D., Estrada, D. \& Pop, E. Low-power switching of phase-change materials with carbon nanotube electrodes. Science 332, 568-570 (2011).

24. Behnam, A. et al. Nanoscale phase change memory with graphene ribbon electrodes. Appl. Phys. Lett. 107, 123508 (2015).

25. Zhu, C. et al. Low-energy phase change memory with graphene confined layer. Appl. Phys. Lett. 108, 252102 (2016).

26. Xiong, F. et al. Towards ultimate scaling limits of phase-change memory. In Proc. IEEE International Electron Devices Meeting 4.1.14.1.4 (IEEE, San Francisco, CA, USA, 2016).

27. Hohenberg, P. \& Kohn, W. Inhomogeneous electron gas. Phys. Rev. 136, B864-B871 (1964).

28. Kohn, W. \& Sham, L. J. Self-consistent equations including exchange and correlation effects. Phys. Rev. 140, A1133-A1138 (1965).

29. Berry, J., Zhou, S., Han, J., Srolovitz, D. J. \& Haataja, M. P. Dynamic phase engineering of bendable transition metal dichalcogenide monolayers. Nano Lett. 17, 2473-2481 (2017).

30. Chandrashekhar, G., Barros, H. \& Honig, J. Heat capacity of $\mathrm{VO}_{2}$ single crystals. Mater. Res. Bull. 8, 369-374 (1973).

31. Qiu, H. et al. The tetragonal-like to rutile structural phase transition in epitaxial $\mathrm{VO}_{2} / \mathrm{TiO}_{2}$ (001) thick films. New J. Phys. 17, 113016 (2015).

32. Togo, A. \& Tanaka, I. First principles phonon calculations in materials science. Scr. Mater. 108, 1-5 (2015).

33. Kiwia, H. L. \& Westrum, E. F. Low-temperature heat capacities of molybdenum diselenide and ditelluride. J. Chem. Thermodyn. 7, 683-691 (1975).

34. Sankar, R. et al. Polymorphic layered $\mathrm{MoTe}_{2}$ from semiconductor, topological insulator, to weyl semimetal. Chem. Mater. 29, 699-707 (2017). 
35. Yalon, E. et al. Energy dissipation in monolayer $\mathrm{MoS}_{2}$ electronics. Nano Lett. 17, 3249-3433 (2017).

36. Yalon, E. et al. Spatially resolved thermometry of resistive memory devices. Sci. Rep. 7, 80 (2017)

37. Kresse, G. \& Furthmüller, J. Efficient iterative schemes for ab initio total-energy calculations using a plane-wave basis set. Phys. Rev. B 54, 11169 (1996).

38. Blöchl, P. E. Projector augmented-wave method. Phys. Rev. B 50, 17953 (1994).

39. Perdew, J. P., Burke, K. \& Ernzerhof, M. Generalized gradient approximation made simple. Phys. Rev. Lett. 77, 3865 (1996).

40. Monkhorst, H. J. \& Pack, J. D. Special points for brillouin-zone integrations. Phys. Rev. B 13, 5188 (1976).

41. Böker, T. et al. Band structure of $\mathrm{MoS}_{2}, \mathrm{MoSe}_{2}$, and $a-\mathrm{MoTe}_{2}$ : angle-resolved photoelectron spectroscopy and ab initio calculations. Phys. Rev. B 64, 235305 (2001).
Open Access This article is licensed under a Creative Commons Attribution 4.0 International License, which permits use, sharing, adaptation, distribution and reproduction in any medium or format, as long as you give appropriate credit to the original author(s) and the source, provide a link to the Creative Commons license, and indicate if changes were made. The images or other third party material in this article are included in the article's Creative Commons license, unless indicated otherwise in a credit line to the material. If material is not included in the article's Creative Commons license and your intended use is not permitted by statutory regulation or exceeds the permitted use, you will need to obtain permission directly from the copyright holder. To view a copy of this license, visit http://creativecommons. org/licenses/by/4.0/.

(c) The Author(s) 2018 